\title{
Rosalba Satalino
}

UNIWERSYTET ŚLĄSKI W KATOWICACH

e-mail: rosalbasatalino@gmail.com

(iD http://orcid.org/0000-0002-0216-8865

\section{La Puglia, una terra da scoprire Un viaggio con Kazimiera Alberti}

\section{Abstract}

\section{Apulia a land to discover. A travel with Kazimiera Alberti}

The goal of this article is to show one of the travelogue of the series Italia celebre e sconosciuta (Famous and unknown Italy) by Kazimiera Alberti: Segreti di Puglia (Apulia's secrets). It is a kind of travel discovering the secrets of Apulian land. The author expresses all the admiration for the landscapes, but above all for the monuments of this region. The history, architecture, myths and legends enrich the pages of her book. She encourages the reader to visit Apulia, revealing only some of her mysteries. Next to Kazimiera Alberti and her husband, we start the exploration of what this land has to offer to the visitors.

Key words: Kazimiera Alberti, Apulia, Italy, travelogue, Segreti di Puglia

Parole chiave: Kazimiera Alberti, Puglia, Italia, reportage di viaggio, Segreti di Puglia 
Nell'ambito letterario dei reportage di viaggio il nome di Kazimiera Alberti è quasi del tutto sconosciuto al pubblico polacco così come a quello italiano. II poco successo delle opere dell'autrice non lo si può attribuire al contenuto degli scritti, ma piuttosto a una poca distribuzione di questi ultimi in entrambi i panorami editoriali. Accostandomi alla letteratura dell'Alberti e alla sua raccolta di travelogue, sono stata particolarmente attratta da un volume, quello dedicato alla mia regione di origine: la Puglia.

L'obiettivo del mio lavoro è proprio quello di presentare al lettore italiano un'opera ancora non sviscerata e analizzata nemmeno dai più esperti studiosi di letteratura di viaggio. Trattandosi di uno scritto poco conosciuto credo che la cosa migliore da fare sia, in prima battuta, quella di esplorare il suo contenuto con la speranza che ciò possa essere un valido punto di partenza per una successiva analisi.

I viaggi della scrittrice e del marito barese attraverso l'Italia, sotto forma di Gran Tour dell'Ottocento, sono stati raccolti dall'autrice stessa in una collana intitolata Italia celebre e sconosciuta. II secondo volume della raccolta, Segreti di Puglia (Alberti 1951), tradotto dal marito dell'Alberti Alfo Cocola e a quest'ultimo dedicato, è stato pubblicato a Napoli dalla casa editrice Conte Stampanel 1951 sotto gli auspici dell'Ente Provinciale per il Turismo di Bari (conta 275 pagine e 60 illustrazioni fuori testo).

Prima di intraprendere il nostro viaggio con l'artista è doveroso spendere qualche parola per spiegare come è composto il resto della collana di reportage di viaggio ambientati nel Bel Paese. Il primo volume, intitolato L'anima della Calabria (1950), è dedicato proprio a questa regione. Fu stampato nel 1950 a Napoli dalla stessa casa editrice del secondo volume e successivamente ristampato nel 2007 dalla casa editrice Rubbettino con la brillante introduzione a cura della professoressa Marta Petrusewicz. La docente definisce l'opera come «una guida-reportage della Calabria - deliziosa, vivace, erudita, ironica e tenera, la migliore che si conosca dopo il classico Old Calabria di Norman Douglas» (Alberti 2007: 5). Vi è anche un reportage dedicato alla Campania, Campania, gran teatro (1953) e uno dedicato alla Liguria, Magia Ligure (1952).

La ristampa del primo volume della serie, L'anima della Calabria, ha spianato la strada alla conoscenza delle opere dell'Alberti da parte del lettore italiano.

All'inizio di questo articolo vorrei sottolineare l'importanza che ha Francesco Giuliani, voce potente ed espressiva e fino a questo momento unica sul territorio italiano, che colloca la scrittrice polacca nella cerchia dei più rilevanti reporter della regione Puglia. Degno di nota è il suo libro Viaggi novecenteschi in terra di Puglia (Giuliani 2009), nel quale unisce tre saggi su tre diversi autori che hanno descritto questa regione nelle loro opere: Nicola Serena di Lapigio e la sua passione per il Gargano, Kazimiera Alberti, un'esule polacca trasferitasi in Italia dopo la seconda 
guerra mondiale e Cersare Brandi, grande scrittore della letteratura di viaggio del Novecento. Tra le pagine di Viaggi novecenteschi in terra di Puglia notiamo una grande ammirazione nei confronti dell'autrice polacca Kazimiera Alberti, definita dallo stesso Giuliani come una scrittrice "colta, sensibile e dalla fertile fantasia» (Giuliani 2009: 121). "L'Italia che scorre sotto i suoi occhi di donna straniera che ha conosciuto il volto più terribile della guerra è varia ed incantevole, ricca di testimonianze illustri del passato come del presente» (Giuliani 2009: 128). Le parole del Giuliani ci dimostrano come questa donna sia in grado di accompagnarci in questa avventura tra le pagine del suo reportage di viaggio e inoltre ne risaltano la capacità di quest'ultima nel trasmettere al lettore, grazie alla sua penna, la sua ammirazione per queste terre tutte da scoprire.

Iniziamo questa esplorazione con Kazimiera Alberti osservando la struttura del libro Segreti di Puglia: partendo da una Prefazione e i primi due capitoli introduttivi il Calderone pugliese e Ave Filia Solis, il testo si suddivide in due parti, nelle quali è possibile distinguere due tipi di itinerari: uno riguardante la zona costiera, da Nord verso Sud, fino a Taranto, che s'intitola La balconata Pugliese; il secondo percorso che ci porta nell'entroterra, la cui prima tappa è Bari, continua prima verso Nord e successivamente scende a Sud, verso il Salento, dal titolo L'interno del calderone pugliese. Proviamo dunque a scoprire come è nata l'idea di questo libro, direttamente attraverso le parole della scrittrice: «La Puglia è stata per me tale una sorpresa che questa da sola ha imposto al libro il nome di Segreti di Puglia» (Alberti 1951: 6).

Sin dalle prime pagine, si instaura un rapporto con il lettore, che è costantemente invitato a visitare e a scoprire i segreti di questa terra. Passo dopo passo l'autrice ci spiega il contenuto del suo libro:

La penna è stata la chiave con la quale ho aperto la porta dei più svariati segreti. Non ho alcuna ambizione di aver spalancato questa porta, e che tutto possa essere visto attraverso di essa. Questo è lavoro e scopo di un uomo di scienze, non di uno scrittore (Alberti 1951: 5).

Seguendo le stesse parole dell'Alberti, vi invito ad intraprendere questa avventura tra le righe della sua piacevole e interessante opera. Nei capitoli introduttivi sottolinea come questa regione non debba avere nulla da invidiare alle altre regioni come la Campania, la Toscana, I'Umbria oppure la Sicilia (Alberti 1951: 7). Infatti, uno dei temi centrali dell'opera è proprio la rivendicazione della bellezza di questa terra, che è riservata solo a turisti dotati di grande intelligenza. Si tratta di una regione in parte non ancora nota alle masse. Proprio nella Prefazione l'autrice evidenzia come nel territorio pugliese ci sia una prevalenza di monumenti da scoprire ed apprezzare (Alberti 1951: 5).

151

Fabrica Litterarum Polono-Italica | 2019, nr 1 (1) 
I temi accennati nella Prefazione iniziano a prendere forma nel primo capitolo, Il calderone pugliese, in cui definisce la Puglia una terra originale che richiede del tempo per poter svelare quello che ha da offrire. È una regione che presenta tracce antiche, a partire dai neolitici che vivevano nelle grotte, che successivamente divennero riparo dei cristiani, poi dimore degli eremiti, ricche di affreschi e segni lasciati da chi ci è passato (Alberti 1951: 7). È come se fosse un riassunto di tutto quello che c'è sul territorio pugliese. Ci spiega, da ogni punto di vista, quello che troveremo in questo calderone.

La presenza di «dolmen» tra le prime forme di espressione religiosa degli esseri umani e di «menhir», una delle più antiche tombe umane, ci fanno capire quanta storia questa terra possa raccontarci. Paragona la Puglia a un calderone che, irradiato dal sole, contiene tracce religiose, filosofiche e culturali di diverse razze (Alberti 1951: 8). Qui il lettore è invitato ad immergersi nel calderone per poter scrutare fino in fondo le bellezze, le ricchezze e i segreti che esso contiene. "Menhir», cattedrali, pergamene, cantine, trattorie, grotte, spiagge e strade dell'antichità, tutte contenute nel calderone "poggiato nell'enorme giardino di ulivi» (Alberti 1951: 11). L'Alberti ci fornisce diverse motivazioni artistiche, paesaggistiche, culturali, folkloristiche per poter visitare la Puglia, pur rischiando di prendersi un'insolazione (Alberti 1951: 12). Un paesaggio non solo da ammirare, ma al tempo stesso da poter assaggiare grazie ai prodotti tipici di questo suolo: olive, uva, mandorle, fichi, ciliegie, prugne, noci, castagne e carrube (Alberti 1951: 11). Sapori che caratterizzano questa terra arida, seppur ricca di prelibatezze da poter assaporare.

Ave, Filia Solis, è tratto da una citazione di Federico II che l'imperatore utilizzò per salutare la città di Brindisi. "Filia Solis» è il nome che l'imperatore svevonormanno attribuì a questa terra non solo per il suo clima, ma anche per la calda cultura che la regione è in grado di offrire (Alberti 1951: 13).

L'autrice descrive i diversi colori del paesaggio durante le stagioni: I'inverno verde, grazie agli alberi sempreverdi come pini, ulivi e fichi d'India; la primavera ricca di mandorli e aranci in fiore e le passeggiate al chiaror di luna che ti danno la possibilità di poter sentire i profumi molto più esotici di quelli di Guerlain (Alberti 1951: 14), esortando così il turista a visitare la Puglia anche in estate andando contro i luoghi comuni che sconsigliavano di visitare questa terra nel periodo più caldo.

Attraverso la descrizione di un'animale tipico della Puglia e del Sud Italia, l'autrice mostra la sua conoscenza del mondo e le sue esperienze di viaggio:

Così altrove ho ammirato i maestosi buoi bianchi dalle enormi corna sullo scenario delle oscure foreste slovacche, i cammelli sulle rosse sabbie del Marocco, della Libia e dell'Egitto [...]. Così ora in Puglia m'incanta il delicato asinello dagli occhi nostalgici che graziosamente risalta tra l'uliveto e la vigna (Alberti 1951: 14).

152

Fabrica Litterarum Polono-Italica | 2019, nr 1 (1) 
La scrittrice crede nel progresso della regione cosiddetta «pietrosa»:

Tra venti anni quando il turista percorrerà questa terra e leggerà in qualche vecchio libro «Puglia pietrosa» certamente sbarrerà gli occhi per la meraviglia vedendosi attorno uno dei più ricchi giardini d'Europa. [...] La Puglia ha cessato di essere "pietrosa», "siticulosa», ma non ha cessato di essere "Filia Solis» (Alberti 1951: 14-15).

Ci presenta una descrizione che coinvolge tutti i sensi: la vista, che ci permette di ammirare i colori; l'olfatto che ci consente di apprezzare i profumi della natura e l'udito che ci appaga i sensi grazie alle canzoni pugliesi un po' arcaiche ed orientali (Alberti 1951: 13-15). In questo viaggio quindi ci lasciamo trasportare dalle sensazioni, dagli odori, dalle splendide viste, dai sapori semplici, seppur ricchi e dai suoni che ci coinvolgono in una tarantella di colori.

Il libro vero e proprio si apre con la sezione La balconata pugliese, formata da 26 capitoli, in cui viaggiamo verso la costa; la seconda invece traccia il sentiero verso le zone interne, L'interno del calderone, con 30 capitoli. Nella prima parte, come accennato precedentemente, il viaggio in compagnia di Kazimiera Alberti e il suo compagno inizia dal Nord verso il Sud, quindi si parte dal Gargano, per poi arrivare a Barletta, Trani, Bisceglie, Bari e come ultima tappa Taranto. Alla fine di questa sezione, nel capitolo conclusivo Ultima scorribanda lungo la balconata, ripercorre il suo viaggio da Nord verso Sud, includendo altre città escluse in precedenza, come Molfetta, Mola, Monopoli, Ostuni e Santa Maria di Leuca, la punta dello stivale. È come se ci volesse dimostrare che ogni volta ci sia la possibilità di poter scoprire altri luoghi e segreti. Ne L'interno del calderone, il viaggio parte dal cuore della regione, Bari, fino ad arrivare al Nord di essa.

Sfogliando le pagine di questo libro possiamo percepire la straordinaria inventiva dell'autrice che, come scrive Francesco Giuliani nel suo saggio, è in grado di rendere diversa ogni pagina, senza perseguire uno schema prestabilito ed è anche d'apprezzare la fantasia che ha nello scegliere i nomi dei capitoli. Originale è poi il modo in cui sono visti il passato e il presente, che permettono di trarre del senso dagli eventi. Molto attenta all'arte e alla cultura, si dimostra una viaggiatrice meticolosa e curiosa di scoprire quello che questa terra è in grado di offrire; una donna che, nonostante il trauma della guerra, nutre speranza e apprezza la bellezza delle cose, descritte con un linguaggio semplice e diretto (Giuliani 2009: 138-140).

Con una descrizione della struttura dell'Italia in Riposo sulla balconata, Kazimiera Alberti descrive la Puglia come una balconata dove ci si può rilassare non solo con la mente, ma anche col corpo esponendo dunque le motivazioni e le aspettative che ha nei confronti di questo viaggio:

153

Fabrica Litterarum Polono-Italica | 2019, nr 1 (1) 
lo riposo moralmente e fisicamente vedendo molti monumenti artistici, vivendo nel mondo dell'arte che mi permette dimenticare tutte le volgarità della vita. [...] lo riposo quando il pensiero respira e lavora calmo e ritmico, anche se senza posa. Sulla balconata pugliese dovrei riposare (Alberti 1951: 20).

Sembra che la Puglia sia il luogo ideale per poter arricchire l'animo e al tempo stesso rilassarsi attraverso la visita di questi monumenti che ci danno la possibilità di non pensare agli episodi negativi della nostra vita e del mondo. È come se questa regione le permettesse di far riprendere la sua anima dopo gli avvenimenti bellici vissuti.

Dopo l'invito al riposo sulla balconata ci addentriamo tra le falde del Gargano e insieme alla scrittrice ci fermiamo alla prima «stazione»: Manfredonia. Arrivati qui, di notte, in pullman, l'Alberti la prima cosa che ci fa notare è che, nonostante sia pieno inverno, c'è un'aria che sa di primavera:

Non aver molta fiducia nel calendario. Anche esso tradisce, falsifica, inganna. Ritarda, avanza, senza motivo. Oggi per esempio, ti comanda di credere che è il $<12$ febbraio $>$. [...] La giornata primaverile ha cancellato l'iscrizione $<12$ febbraio $>$ e si fa beffe del calendario (Alberti 1951: 21).

Ci descrive i paesaggi e la natura, colei che sa essere crudele e al tempo stesso stupefacente. La città, definita dall'autrice una giovane sposa (Alberti 1951: 22), prende il posto di Siponto. Fa riferimento all'epoca degli Angiò e ricorda Giacoma Beccarini, la donna strappata alla sua terra nativa dalla corte di Costantinopoli, che da schiava divenne sultana turca. Il turchese, colore del mare e del cielo, domina le righe di queste pagine e sembrano donare all'autrice un senso di calma e tranquillità (Alberti 1951: 21-24).

In questo tragitto ci svela come le falde garganiche siano ricche di segreti, di alberi spogli che formano lettere ebraiche sulle terrazze, ma all'improvviso tutto ciò si interrompe di fronte alla visione di un albero in fiore. L'eco della guerra mondiale si fa spazio tra le righe e la metafora dell'albero in fiore rende esattamente l'idea della possibilità di poter rinascere anche dopo un lungo periodo di buio:

[...] l'albero in fiore, simbolo di miracolo. Dagli scuri, secchi rami spuntano i primi boccioli, a decina, a centinaia [...]. II secco scheletro dell'albero risorge. Vi è in questa trasformazione qualche promessa per l'uomo. Gli occhi han visto il crimine del secondo uragano mondiale vedono d'improvviso... un albero in fiore (Alberti 1951: 24).

154

Fabrica Litterarum Polono-Italica | 2019, nr 1 (1) 
Non è un viaggio solo alla scoperta del territorio pugliese, poiché grazie alle bellezze che ci sono è un percorso che permette al proprio animo di riprendersi, di rimarginare le ferite, nonostante i ricordi dolenti del passato.

Restiamo ancora un po' nello Sperone d'Italia, il Gargano, tuttavia l'autrice ci porta sulla sua cima più alta, laddove si trova l'antenna garganica. II Monte Sant'Angelo, luogo di apparizioni, nel passato una delle tappe fisse durante i pellegrinaggi, diventa il protagonista insieme ai racconti legati alla leggenda dell'Arcangelo Michele (Alberti 1951: 25-27). Come è possibile osservare dalle parole di Giuliani, in queste pagine si nota la formazione laica della scrittrice polacca, che utilizza un percorso descrittivo personalizzato (Giuliani 2009: 142).

Il campanile alletta l'immaginario della scrittrice e perciò ai suoi occhi diventa una sorta di antenna che collega la grotta e il cielo, abbattendo qualsiasi barriera, comunicando i diversi messaggi di pace di tutto il mondo (Alberti 1951: 27).

Sotto le ali dell'Arcangelo giungiamo fino alla grotta di Monte Sant'Angelo, dove l'eco della guerra rimbomba come un grande frastuono attraverso le parole dell'Alberti. Lo scendere le scale le riporta in mente il momento in cui si rifugiava in cantina dopo aver udito il suono dell'allarme bomba (Alberti 1951: 29). Le ali sotto le quali si ritrovano i due coniugi sono quelle della <Porta alata> della Basilica di Monte Sant'Angelo, quelle della statua dell'Arcangelo, eppure il desiderio dell'autrice è il poter creare delle ali, che ci permettano di rimettere a nuovo le nostre vite (Alberti 1951: 28-30). È interessante come non si soffermi sulla descrizione dei pellegrinaggi, ma piuttosto decida di presentarci i dettagli della statua dell'Arcangelo.

Successivamente, Kazimiera Alberti ci propone uno dei segreti pugliesi per antonomasia: l'enigma della Tomba di Rotari. L'invito che ci porge in questo caso è quello di provare a risolvere diversi rebus: la funzione del monumento, il quadrato che non sembra un quadrato, i capitelli, delle scale che non portano da nessuna parte e così via... (Alberti 1951: 30-33).

Cerca di smorzare i luoghi comuni creati sulla gente del Sud Italia:

[La] gente [ha] una certa gentilezza e socievolezza, ed un più dolce modo d'agire e di ospitalità. II pugliese che in genere è abbastanza arido e non fraternizza molto, a Monte Sant'Angelo si è mutato in un uomo piacevole e pieno di cortesia (Alberti 1951: 35).

Non mancano qui descrizioni dei monumenti e anche una riflessione sulla morte dello studioso Giovanni Tancredi, lodato dall'Alberti perché era stato in grado di promuovere al meglio la sua terra, il Gargano. Qui possiamo condividere con la coppia un episodio ricco di folklore, che appaga le aspettative dell'autrice polacca, mangiando del pane e bevendo del vino in quel di Monte Sant'Angelo (Alberti 1951: 33-37).

155

Fabrica Litterarum Polono-Italica | 2019, nr 1 (1) 
Dopo aver riempito i nostri animi con racconti legati a figure religiose, aver provato a risolvere enigmi e ad esserci saziati con un pasto semplice e genuino ci spostiamo verso la prossima "fermata», una piccola città ricca di meraviglie: Barletta, una località famosa per il suo vino e per i suoi monumenti. È una cittadina che stuzzica tanto la curiosità della scrittrice poiché in essa si concentrano tante storie e testimonianze (Alberti 1951: 38-41).

Il tragitto per raggiungere la prossima sosta non è molto lungo, considerando che la nostra prossima "stazione» è proprio Trani. Ci dona una descrizione di questa località da tutte le angolazioni. La cosa che possiamo notare sin da subito e che più ci intriga è il paragone di quest'ultima ad una donna:

Molte donne colpiscono subito, ci incantano e ci legano a loro al primo colpo d'occhi; ci seducono immediatamente. Altre hanno bisogno di una più profonda conoscenza per mostrarci le loro attrattive e piacerci. Lo stesso avviene per le città. Trani è una cittadina tanto pittoresca, linda, quasi civettuola nel suo fascino, che ci attira e guadagna le nostre simpatie subito, dal primo giorno di conoscenza (Alberti 1951: 42).

Una città che l'ha colpita sin da subito, dal primo momento, tanto da farle scrivere un'interessante descrizione di essa, con un tocco di poesia. Non c'è ammirazione solo nei confronti di Trani, ma un vero e proprio elogio alla Penisola Appenninica, che è vista dall'autrice non solo come una miniera infinita per archeologi, ma anche per gli psicologi, poiché grazie alla storia, mitologia, filosofia e alla bellezza naturale molti artisti, santi, filosofi, politici ecc. I'hanno scelta come luogo di riparo. Ne descrive paesaggi, monumenti, storia e non solo, in quanto parla anche di un filosofo e politico, Giovanni Bovio, cittadino di Trani, che ha tanto combattuto per la giustizia, che non sempre è stata onesta (Alberti 1951: 42-45).

La prossima tappa ci porta ad esplorare un'altra cittadina, Bisceglie, ma al tempo stesso ci fa fare un viaggio indietro nel tempo, portandoci fino ai tempi della preistoria. Ricco di domande sulla filosofia umana, questa volta l'invito che l'autrice ci pone è quello di avvicinarci a guardare questa prima pagina scritta dall'uomo primordiale, il dolmen:

Turista blasè! Non voglio suggerirti che forse ti trovi in un luogo sacro. In ogni caso questo è certo non è un banale angolo della terra né un posto tanto facile da incontrare. Guarda questo primo documento di pietra nel quale l'emotivo troglodita, mio, tuo, nostro antenato, ha annotato tutte le sue nostalgie spirituali e morali. Guarda questa prima pagina del pietroso libro sulla quale egli ha scritto la prima «A» della civiltà (Alberti 1951: 47).

156

Fabrica Litterarum Polono-Italica | 2019, nr 1 (1) 
Ritorna alla preistoria, facendo riferimento alla conoscenza universale e definendo I'Italia un'enciclopedia vivente (Alberti 1951: 46), in cui si possono scoprire diverse curiosità, solo se si è predisposti alla conoscenza, altrimenti «se sei solo uno "sfaticato" conoscerai soltanto... "i maccheroni" e "O sole mio"» (Alberti 1951: 46). Ribadisce quindi il concetto che questa sia una regione per turisti perspicaci. Attraverso il "dolmen» di Bisceglie, inizia una riflessione sulla morte e la filosofia primordiale. Questa pietra manteneva un contatto con la divinità che proteggeva ed accarezzava il defunto. Ancora una volta, esorta il viaggiatore a «scoprire questo primo monumento della civiltà umana che ha superato millenni, terremoti e cicloni» (Alberti 1951: 48). Il dolmen è la dimostrazione di come l'uomo abbia vinto per la prima volta contro l'inerzia della materia, laddove nacque la tecnica, lì dove tutto ebbe inizio. Questo momento è paragonabile alle fondamenta dell'impero della civiltà (Alberti 1951: 49). Ai piedi del dolmen sorgono degli interrogativi umani ai quali probabilmente non sono ancora state trovate delle risposte.

Dalla preistoria facciamo un salto al ricovero ultra moderno. Siamo sempre a Bisceglie ed il viaggio prosegue con una guida Saverio Majellaro (al quale è dedicato il capitolo), ponendo l'attenzione sulle grotte, donandoci una descrizione minuziosa, suggestioni e riflessioni sui nostri antenati; tuttavia questo luogo inevitabilmente porta la scrittrice a pensare ai bunker e ai rifugi sotterranei, nei quali era stata costretta a rintanarsi quando l'allarme cominciava a suonare: "Mai la grotta è stata modello di architettura tanto attuale come nel XX secolo!» (Alberti 1951: 52).

L'album biscegliese è lì dove presente e passato si incontrano e dove ogni dettaglio di questa città viene esaltato dall'autrice. Ricca di particolari, la descrizione vivace ci illustra ogni angolo della cittadina: dal magazzino al laboratorio, alla via fino ad arrivare alle arcate. In questo album ritroviamo anche le caratteristiche tipiche delle città del meridione. Quelle località in cui è necessario vedere non solo l'interno dell'abitazione del cittadino, ma anche e soprattutto l'esterno:

La via [...] è l'arena sulla quale già si affaccia il bimbo di un anno a fare la sua prima conoscenza con la casa di pietra, coperta dal tetto il più bellamente colorato del mondo, il cielo d'Italia (Alberti 1951: 56).

Sulla strada la donna ci sorride e gioca con l'infante, ripara le calze, fa la siesta, pettegola con le vicine... Ma ecco che alla fine lei e lui son diventati vecchi. Egli porta la sedia davanti la casa e riposa. Ha gli occhiali sul naso e legge la gazzetta. Lei sbuccia fave, piselli o castagne. Han finito di essere gli attori dei vicoli e piazzette. Hanno preso ora il ruolo di spettatori (Alberti 1951: 57).

Queste sono le tradizioni che, nonostante la città si modernizzi, restano radicate per generazioni e generazioni. Non mancano in questo capitolo, i confronti con le altre nazioni: "L'inglese ama la sua "Home" ed il campo di sport, [...] il Tedesco la cucina; I'Italiano ama soprattutto la strada» (Alberti 1951: 58). 
L'album biscegliese è il riassunto della vita quotidiana a Bisceglie, ne descrive le strade, le case, la gente. Per poter vedere esattamente questo quadro della quotidianità locale l'unico elemento sul quale basta soffermarsi per interpretarla a pieno è la strada. Perché è proprio lì che l'italiano trascorre le sue giornate, lavora, vive, prega e così via (Alberti 1951: 58).

Conclusasi la presentazione di Bisceglie, all'improvviso ci ritroviamo di fronte ad «un incontro amoroso» con Barion, il fondatore del capoluogo pugliese.

Le pagine sono attraversate da un tocco di fantasia, di brio che ci pervade sin dalle prime righe con un bigliettino ricevuto dall'autrice, da un certo Barion, che le dà appuntamento alla stazione di Bari (Alberti 1951: 59). Egli si presenta come un uomo elegante e distinto che ha fondato la città di Bari e ha deciso di guidare l'Alberti tra le vie della sua cittadina. La scrittrice, incuriosita, inizia a fare diverse domande sull'identità di questa figura, che si presenta come un fondatore della città, che ha un età "sottointesa» (Alberti 1951: 59). Quest'ultimo, che accompagna l'autrice in questa visita, porta alla luce anche eventi del passato: guerre, epidemie, cataclismi della natura. Tuttavia lui era sempre pronto a salvare la sua città e a ricostruirla pezzo per pezzo. Kazimiera Alberti è completamente ipnotizzata da questo "uomo» e dai suoi valori (Alberti 1951: 59-61).

In questo dialogo un po' bizzarro, il Barion chiede all'autrice polacca di guidarlo per la visita della città: da guida.

- Oggi sono rivenuto per vedere questa città nuova. Vi prego di farmi

- Io!? Ma io sono una straniera, non lo sapete?

- Lo so benissimo! Appunto per questo vi prego. Lo straniero vede sempre le cose caratteristiche (Alberti 1951: 62).

Questo dialogo continua, toccando elementi come l'acqua, la fontana, l'università, il lungomare, la pinacoteca provinciale. Anche l'esaltazione degli uomini vitali pugliesi è qui sottolineata, sempre per poter poi criticare quegli uomini che utilizzano la propria scienza per organizzare le guerre (Alberti 1951: 63).

Nel momento in cui si parla della Fiera del Levante, c'è un elogio ai baresi che con le loro iniziative e capacità organizzative sono stati in grado di creare questa fiera. Nella fiera Barion è contento, adora la folla chiassosa, si diverte come un ragazzo (Alberti 1951: 66-67).

Questo romantico appuntamento con Barion termina dopo una bella giornata con il chiarore della luna e un mazzo di garofani rossi che il fondatore regala all'Alberti:

Barion si allontana un istante. Ritorna con un fascio di lunghi garofani rossi. - Per il vostro disturbo cicerone... 
Avvicino i garofani alle narici. Di colpo il posto a me di fronte è vuoto. Barion è sparito. Come una visione, come una nebbia, come un'onda (Alberti 1951: 67).

Questa invenzione narrativa cattura l'attenzione del lettore, che si fa coinvolgere da questo flirt intellettuale che la donna ha con Barion. Pur non dedicando una sezione a parte al capoluogo pugliese, le doti artistiche dell'Alberti ci fanno vivere la descrizione di Bari con un po' di freschezza e di vivacità.

Con grande abilità e maestria la nostra "cicerone» ci mostra le due medaglie sulla storia del santo patrono della «porta d'Oriente»: San Nicola. Ci offre un quadro della visione che i bimbi polacchi hanno di quest'ultimo: tra il 5 e il 6 dicembre, egli porta i doni ai bambini, come la Befana in Italia porta i doni ed i dolcetti il 6 gennaio. Oltre ad essere il santo adorato dai più piccoli, San Nicola è anche il santo adorato dagli innamorati. Ai bambini polacchi più poveri giungevano i regali sotto il guanciale, grazie ad associazioni e comitati del posto (Alberti 1951: 69). Questo dimostra come la figura di San Nicola sia davvero importante per il popolo polacco.

L'immagine che l'autrice ha avuto di San Nicola è appunto quella che tutti i polacchi hanno sempre avuto, ovvero di colui che porta i doni ai bambini e agli innamorati. Eppure una volta arrivata a Bari, questa figura viene stravolta. Ora ha di fronte a sé un santo che protegge i marinai dalle catastrofi. Si rende conto che l'altra "fotografia» di San Nicola sia sconosciuta agli occhi dei baresi. Difatti nel capoluogo pugliese ogni anno il 9 maggio si rende omaggio al santo, che arrivò da Mira via mare, circondato da 42 marinai. Per Kazimiera Alberti, come per tutti coloro i quali venerano San Nicola come quella figura che porta i doni, rileggendo la sua storia, è possibile comprendere che in realtà è colui che raccoglie, colui che aiuta (Alberti 1951: 68-70).

In questa sosta nel capoluogo pugliese, la scrittrice polacca ci parla della Basilica di San Nicola di Bari e della leggenda di San Nicola. I monumenti, uno dei temi ricorrenti nel libro, soprattutto quelli romanici come in questo caso la Basilica di San Nicola, intimoriscono i fedeli portandoli a riflettere che non sono necessari il lusso e l'ostentazione della ricchezza, ma che la semplicità e le rinunce possono riempirci il cuore e l'anima e portarci ad un esame di coscienza (Alberti 1951: 74).

L'Alberti racconta come i baresi rubarono le sacre reliquie del santo da Mira, che nel 1087 giunsero nel porto di Bari su una nave mercantile. La basilica venne costruita appositamente per poter depositare le reliquie di San Nicola, però l'autrice non si sofferma sui vari cambiamenti esterni avvenuti nel corso dei secoli, ma sulla psicologia, sul pensiero, sulla spiritualità ad essa legata (Alberti 1951: 71-74).

Attraverso un'interessante metafora, ci presenta l'anima della «porta d'Oriente» - «Bari Vecchia»: 
Ogni città ha le sue curiosità! Bari, ha il suo magnifico vaso. Tu domanderai subito, con interesse: «E qual è questo vaso?». [...] Si chiama «Bari Vecchia» (Alberti 1951: 75).

[...] In questa isola a forma di vaso puoi entrare con facilità da ogni lato, ma I’uscirne è cosa ben più difficile (Alberti 1951: 76).

Nel corso dei secoli qui tutto è rimasto invariato, i gesti quotidiani sono ripetuti nel tempo, l'artista che canta con la sua chitarra, l'artigiano che lavora davanti alla sua casa, il mercato in miniatura, l'asinello che trasporta la merce e così via. È un teatro ricco di sfaccettature, una diversa dall'altra, Bari vecchia rappresenta la vita (Alberti 1951: 77-78). Possiamo dunque perderci per le vie del centro storico di Bari assaporando a pieno lo spettacolo folkloristico che solo il capoluogo pugliese è in grado di offrirci.

Dopo esserci goduti lo show a cielo aperto di Bari vecchia, iniziamo un nuovo viaggio nel tempo con un orologio che non segna solo le ore, i minuti e i secondi, anzi ci consente di fare un salto addirittura nell'antichità. L'arrivo della fiamma olimpica nel capoluogo della regione, per i giochi olimpici di Londra del 1948, ispira l'Alberti, che inizia un excursus a partire dalle origini delle olimpiadi nell'antica Grecia, descrivendone le abitudini collegate ad essi, fino a giungere alla versione moderna. Dopo la pausa dovuta alla guerra, la scrittrice esalta i valori pacifici di questa manifestazione, che finalmente superano l'oscurità creata degli eventi bellici (Alberti 1951: 79-85). Ricorre ancora una volta la ricerca di pace e serenità, un desiderio di tregua, di tranquillità dopo il subbuglio e il caos creato dalla guerra.

Spostandoci verso Sud durante la nostra esplorazione della Puglia, giungiamo a Egnazia, città nota per la sua ceramica e per essere stata menzionata in una satira di Orazio. Questa città venne distrutta da Totila nel VI secolo e anche in questo caso l'autrice non rinuncia a parlare in chiave negativa dell'uomo che distrugge:

Ma l'uomo-Totila, il crudele concorrente degli elementi infuriati, non è mutato. Ed è lo stesso se Egnazia si chiami Varsavia od Amburgo, Rotterdam o Colonia, Belgrado o Cassino, Nagasaki o Stalingrado. Tutto eguale, lo stesso! Si svolga nel 545 o nel 1945... (Alberti 1951: 88).

Il rimbombo della guerra irrompe ancora una volta nel testo. Questo vagabondare ci porta a fare un esame di coscienza. Ritrovare ogni volta la forza di ricostruire una città, per ricominciare, non è semplice. Infatti il capitolo si chiude con una domanda dell'autrice:

Ma domani se dovessero ripetersi molte Nagasaki su ogni dimensione geografica, [...] avremo più la volontà di ritornare sulle rovine e ricostruire di nuovo? (Alberti 1951: 88). 
Attraverso l'antica "Traiana» ci dirigiamo insieme alla coppia Tra le corna del cervo, a Brindisi. L'autrice polacca qui delinea il paesaggio di questa tratta, tra gli ulivi e le loro stravaganti forme, "simbolo della pace» e "albero sacro del Mediterraneo" (Alberti 1951: 89-90). Brentesion fu creata all'epoca cretese, in questo punto: tra le corna del cervo. Ci spiega come il nome di questa città derivi appunto dalla parola messapica brunda ovvero cervo per la forma dell'insenatura che ci fa pensare ad una testa di quest'ultimo. Notevole anche la spiegazione della differenza tra il significato delle corna del cervo in due diverse discipline: la zoologia e la geografia. Nella prima simboleggiano inquietudine, combattimento; nella seconda rappresentano un posto molto calmo (Alberti 1951: 90-91). Ancora una volta dimostra la costante ricerca di tranquillità dell'autrice, colmata dalla serenità trasmessa da questa terra.

La prossima fermata, la terra di mezzo tra Oriente e Occidente sin dai tempi più remoti, è Otranto. La storia è il centro della narrazione. Il tallone dello stivale d'Italia (Alberti 1951: 94) è testimone di una delle tragedie religiose che hanno coinvolto migliaia di innocenti, per lo scontro tra i Cristiani e i Musulmani. II massacro avvenuto nel 1480 ha segnato profondamente la storia di questa cittadina che però nonostante tutto è sempre lì, lungo la sua costa scaldata dal sole (Alberti 1951: 98). In qualche modo l'autrice cerca sempre di trovare uno spiraglio di luce alla fine del tunnel.

In questa sosta ad Otranto, l'Alberti ci porta a vedere da vicino il pavimento in mosaico della Cattedrale opera del prete Pantaleone, minuziosamente descritto, che ha lasciato a bocca aperta la scrittrice e alletta il lettore alla scoperta di questa meraviglia. Si tratta di una città, Otranto, che è stata segnata da diversi avvenimenti storici turbolenti, nella quale è presente un senso di quiete, che però è nato dopo aver subito eventi negativi. I misteri tuttavia non sono ancora terminati. In questa cittadina c'è un altro segreto pugliese legato a una torre romana, un tempo faro, che si spegneva all'improvviso. Il ladro dell'olio del faro si dice che fosse un serpente marino, attualmente disegnato nello stemma della città (Alberti 1951: 102). Lava indurita ad Otranto è il frutto di una riflessione nata sulle località che hanno qualcosa da raccontare: "Sono le città stigmatizzate a fuoco, e la loro temperatura "sotto-pelle" le distingue spesso dalle grandi, fredde metropoli» (Alberti 1951: 102).

Ritorniamo ai preziosi insegnamenti dalla preistoria in Lezione di geologia nella grotta Romanelli. Questa lezione di geologia è svolta dalla balconata pugliese che gli ha permesso di ammirare come è formato il territorio. A Castro, l'Alberti e Cocola hanno la possibilità di fare un'escursione in barca che gli permette di raggiungere la grotta Romanelli, nascosta tra «i resti delle zanne di "elephas antiquus" ॥ (Alberti 1951: 103).

L'autrice illustra i dettagli di questa grotta, che si riesce a dedurre fosse prima sotto il livello del mare e ci svela attraverso la descrizione dei segni che ci sono

161

Fabrica Litterarum Polono-Italica | 2019, nr 1 (1) 
sul pavimento, la presenza di testimonianze dei periodi interglaciali (Alberti 1951: 104). Invita dunque il lettore a fare questa magnifica passeggiata in barca per poter assaporare ed immergersi in questo spettacolo della natura.

II monumento particolare di Patù (un piccolo paesino in provincia di Lecce) soprannominato "Le Centopietre» è il protagonista de L'ignoto testimone di un celebre incontro. Il celebre incontro è quello fra due culture differenti che si unirono per poter creare questo monumento dedicato a Dio:

"Le Centopietre» (poiché sono giusto cento i blocchi di pietra squadrata che lo costituiscono), ha un aspetto dimesso, modesto; direi quasi che filosoficamente voglia dall'alto del suo sangue nobile ridere di ogni pompa esteriore. Ma nella sua strana veste di baracca litica forma un altro importante anello della grande catena dello sviluppo dell'umanità (Alberti 1951: 108).

Viaggiando sempre nel Salento, dopo aver raggiunto Gallipoli sulla costa ionica, la scrittrice ne fa un'accurata descrizione ne Il pesce sul piatto di Maiolica. Per esaltare la bellezza della città scrive:

O uomo del nord, se tu sogni del sud, di un lido azzurro sul quale riposare, ti auguro ti appaia nel sogno una cittadina situata sotto il tacco dello stivale italiano: Gallipoli! Già il suo nome esotico ti dirà che è «Bella città». "Kalli-polis» la chiamarono i suoi padrini di battesimo, i Greci (Alberti 1951: 110).

Tutta la descrizione ruota attorno al paragone della città con un qualche "pesce antidiluviano, emerso dal mare per riposare, che incantato dal colore non è più ritornato alle sue profondità" (Alberti 1951: 110). Poiché:

Quando il vento si calma, il mare, sul quale posa l'enorme pesce dalla testa tagliata, si presenta come un piatto di lucida maiolica, cosparso di tutte le sfumature di azzurro, dal più chiaro al più cupo (Alberti 1951: 110).

Ci facciamo travolgere dalla bellezza dei monumenti della graziosa città a partire dalla Fontana Ellenica fino ad arrivare alla Cattedrale. Ci ritroviamo così coinvolti in una riflessione sulla giovinezza che porta all'esaltazione della poesia, in grado di ringiovanire le cose, e alla critica dell'archeologia, che tende solo ad invecchiarle. La visita di questa località è accompagnata da un'orchestra, i cui protagonisti principali sono le onde ed il vento. Il castello angioino che contiene segreti storici, a ridosso del mare; la cattedrale in stile barocco, seppur meno sfarzoso rispetto allo stile di Lecce, ogni cosa riguardante questa cittadina allegra, laddove ci si possono compiere diversi peccati anche in un unico giorno, trova un piccolo spazio per essere descritto tra queste pagine (Alberti 1951: 111-115).

162

Fabrica Litterarum Polono-Italica | 2019, nr 1 (1) 
Attraverso le note del vento e del mare ci lasciamo trasportare lungo il cammino verso Taranto per Un'amichevole visita a Taras. In questa nuova tappa l'autrice fa di nuovo ricorso ad una figura leggendaria, come avvenuto in precedenza per il capoluogo pugliese, colui che ha fondato il capoluogo ionico: Taras, il figlio di Nettuno ed il suo delfino. La ricchezza di questa città per l'Alberti è proprio il mare, che ha dato tutto a questa popolazione, talvolta anche la morte (Alberti 1951: 116). Tra i prodotti tipici del posto, spiccano i frutti di mare, che possono divenire «un soggetto meraviglioso per un pittore di nature morte» (Alberti 1951: 117).

Gli odori, i profumi, i frutti di mare, il clima, le portano alla mente Marsiglia, la Spagna, la Grecia. Le fanno ricordare l'aria che si respirava nel Mediterraneo (Alberti 1951: 117). Non si sofferma solo sulla natura e il paesaggio, quindi decide di recarsi in visita al museo, il cui proprietario, Ciro Drago (al quale dedica il capitolo), le dà la possibilità di visitarlo, pur essendo ancora chiuso al pubblico dopo la seconda guerra mondiale. La sua sete di conoscenza la porta nel museo che le permette di immergersi e perdersi tra i reperti della Magna Grecia (Alberti 1951: 118).

Ancora una volta con un tocco di fantasia ci si ritrova in compagnia del fondatore della città a bere del vino locale. L'incontro si conclude con un invito a fare un tuffo nel passato sul dorso del delfino di Taras (Alberti 1951: 120). Di qui inizia un excursus sulla storia di Taranto, "una stella della Magna Grecia» (Alberti 1951: 121), dalle origini alle vicende di Taras fino ai tempi di Annibale per risaltarne l'importanza nel corso dei secoli.

Nell'Ultima scorribanda lungo la balconata, poi, ripercorriamo con l'autrice tutto il suo itinerario, con qualche tappa in più. Ripartiamo ancora una volta dal Gargano con l'esaltazione dei paesaggi che le scorrono davanti agli occhi: Rodi, Vieste, Manfredonia (Alberti 1951: 127). Poi una sosta alla stazione termale, Margherita di Savoia, definita dall'autrice «la più giovane città della balconata» (Alberti 1951: 127), che prese il suo nome solo settant'anni prima della visita della scrittrice. Facciamo un salto a Molfetta, per poter ammirare la cattedrale di San Corrado, che colpisce la donna nonostante ne abbia viste diverse e di stupende. Quello che la incanta è lo stile locale delle tre cupole per le quali è stata utilizzata la «copertura piramidale dei trulli» (Alberti 1951: 127).

Lungo la costa facciamo un'altra piccola fermata a Mola di Bari per poter gustare con la coppia del vino e del pesce fritto che lasciano in loro un senso di leggerezza e spensieratezza:

[...] la gastronomia polacca afferma che «dopo pesce e vino l'uomo è leggero come una piuma, dopo carne e birra è pesante come un legno» (Alberti 1951:130).

Con la pancia piena e un po' di serenità ci prepariamo ad ammirare Polignano a Mare: in particolare quello che salta all'occhio dell'autrice è la grotta Palazzese,

163

Fabrica Litterarum Polono-Italica | 2019, nr 1 (1) 
con i suoi colori che passano dall'argento al platino, da toni grigi a toni verdastri (Alberti 1951: 130). Colori che ci riempiono l'animo e ci permettono di riposarci, rinfrescarci.

Siamo pronti per la nostra prossima stazione definita dall'Alberti una tra le poche città industriali della regione, Monopoli, la "Città Unica" (Alberti 1951: 131).

Ne ammira la Cattedrale e si sofferma sulla curiosa leggenda che si cela per la costruzione del tetto di quest'ultima: la Cattedrale, costruita su richiesta del vescovo Romualdo, non poteva essere completata per la mancanza delle travi necessarie per il tetto; durante la notte del 16 dicembre 1117, il vescovo ebbe una visione e dopo essersi recato a "cala batteria», il porticciolo della città, scoprì in mezzo al mare una zattera costruita con tavole di cedro, sulla quale c'era il quadro della Madonna della Madia, attuale Santo Patrono della città. Grazie a quelle travi è stato possibile finire la costruzione della chiesa (Alberti 1951: 131).

Come suggerisce la stessa autrice:

Forse non a caso la balconata ha approfittato proprio di questo panorama moderno di fabbriche, serbatoi e ciminiere per inquadrarvi uno dei suoi più romantici racconti (Alberti 1951: 132).

Ultima tappa del viaggio lungo la balconata pugliese è Santa Maria di Leuca: de Finibus Terrae. Un tono malinconico pervade le righe che descrivono questo luogo, poiché porta Kazimiera Alberti a riflettere sul fatto che ogni cosa abbia un inizio ed una fine:

Qui finisce la Terra! Finibus Terrae!

Un posto malinconico, sul balcone pugliese! Ci ricorda che tutto ha una fine. Che la vita comincia con l'innocente Mattinata garganica e finisce con la oscura sera a S. Maria de Finibus Terrae (Alberti 1951: 133).

Tanti simboli ci accompagnano in quest'ultima scorribanda lungo la balconata: il sole con cui il viaggio inizia e il buio con cui termina, metafora della vita; la partenza dal Gargano al nord, fino ad arrivare giù nelle acque lontane a Santa Maria de Finibus Terrae, dove il mare infinito ci porta a pensare all'eternità. La rosa che accompagnerà l'Alberti durante il viaggio sarà infine gettata tra le onde:

Siamo partiti al mattino presto e la nostra rosa ottobrina era in bocciolo. A mezzogiorno nella calda Mola era in pieno rigoglio. Ora che s'inizia il tramonto è appassita. [...] La rosa è sfiorita! La gettiamo alle onde! Non sappiamo verso dove navigherà! [...] Quando arriverà la sera della vita qualche mano getterà anche noi alle onde (Alberti 1951: 132-133).

164

Fabrica Litterarum Polono-Italica | 2019, nr 1 (1) 
Le fasi del giorno e l'aspetto della rosa, che cambiano man mano con il passare delle ore, sembrano voler simboleggiare le fasi della vita: la giovinezza rappresentata dal bocciolo al mattino, il pieno della vita durante l'età adulta a mezzogiorno e al tramonto, quando la rosa è ormai appassita, giungiamo alla vecchiaia. Ormai sfiorita, la rosa, viene gettata tra le onde verso l'infinto e l'eternità del mare.

Con questa riflessione sulla vita e la rosa trasportata chissà dove dalle onde, ci prepariamo ad allontanarci dal mare per arrivare nelle viscere del calderone pugliese. Siamo pronti ad esplorare la Puglia dall'entroterra nella seconda sezione L'interno del calderone. La novità in questo caso non è solo il percorso che prende un'altra direzione, ma anche il mezzo di trasporto che l'Alberti utilizza per spostarsi: il treno. Dai finestrini delle "Ferrovie del Sud-Est" tornano a galla i ricordi della scrittrice legati a questo mezzo di locomozione, il treno, che l'ha portata in diversi posti del mondo. La ferrovia del Sud-Est viene elogiata da Kazimiera Alberti, non solo per la sua funzione economica, ma piuttosto per la sua funzione culturale; utilizzava il treno per tratte ben più lunghe, per esplorare nuove terre e non per uso domestico. Difatti descrive la ferrovia del Sud-Est in maniera entusiastica, per dimostrare che la Puglia era riuscita a progredire per quanto riguarda i mezzi di trasporto:

Nelle vecchie guide leggiamo che "La Puglia è difficile a visitare per la mancanza di mezzi comodi di locomozione» (Alberti 1951: 140). [...] nessuno può già più dire che la Puglia sia regione difficile a visitare (Alberti 1951: 142).

Elogia le ferrovie che permettono di visitare anche i paesini più piccoli, di ammirare paesaggi fantastici attraverso il finestrino ferroviario (Alberti 1951: 142).

La prossima stazione è quella di Modugno, la cui narrazione ruota attorno al rapporto tra l'uomo e il sacro. L'Alberti, essendo una viaggiatrice, nel corso dei suoi viaggi ha avuto la possibilità di «incontrare» diverse divinità di differenti culture:

In Egitto, con diffidenza mi avevan guardato gli occhi eterni di Osiride. [...] Al Vaticano lo Zeus di Otricoli chiudeva nei suoi occhi, come un libro prezioso, tutta la filosofia dell'antichità. Sulle rive della Vistola vidi Swiatowid, il Dio che vede il mondo intero. Aveva quattro facce, volte ai quattro angoli del mondo. [...] In Grecia, a Dafnae, avevo ammirato il Dio bizantino, cupido della potestà della terra. [...] Da Costantinopoli al Marocco il Dio dell'Islam [...]. In Francia ed in Spagna, in Olanda ed in Belgio, il Dio Cristiano, rappresentato in migliaia di magnifiche edizioni [...] (Alberti 1951: 144).

Nonostante avesse avuto l'opportunità di ammirare diversi esseri divini in giro per il mondo, qui, a Modugno, la scrittrice resta sorpresa nel vedere una divinità diversa dal solito, eppure con due caratteristiche tipiche: "la impenetrabile indifferenza e lo splendido mutismo» (Alberti 1951: 145). Questa divinità coincide con

165

Fabrica Litterarum Polono-Italica | 2019, nr 1 (1) 
il Menhir di pietra, soprannominato il Monaco (Alberti 1951: 145). Per Kazimiera Alberti il Monaco è la prima divinità assoluta ad aver avuto una forma, una sorta di mentore per gli esseri divini che a suo dire siano comparsi successivamente e in ambiti più specifici. Ai piedi di questo monumento neolitico, inizia una riflessione sulla vita e traspare la sua formazione laica con un tono quasi critico nei confronti di coloro che si rivolgono a entità superiori con le loro domande:

Siedi ai suoi piedi. Non chiedere nulla perché nulla ti risponderebbe. Nulla guadagnerai a supplicarlo. Alcun peccato egli ti rimetterà. Né t'imporrà penitenze. Né ti darà premio alcuno! Non ti consiglierà niente. Non ti profetizzerà nulla. Non ti destinerà a nulla. Non ti obbligherà con alcun codice (Alberti 1951: 146).

Restiamo ancora ne // mistico triangolo modugnese, accanto al Menhir neolitico, lì dove c'è la chiesa di San Pietro di Basignano, una tra le più antiche chiese romaniche pugliesi, con la sua cupola che raffigura il cielo e la grotta dove ha vissuto San Corrado, in cui il santo ha cercato di creare una comunione con la Divinità. II triangolo spirituale è dunque composto dal Menhir, dalla chiesa di San Pietro e dalla grotta di San Corrado (Alberti 1951: 148-150).

Dopo questa sosta a Modugno, accompagnata da una riflessione sulle divinità e il sacro, la nostra "cicerone» ci invita a soffermarci ad osservare una piccola località quasi sconosciuta: Bitonto. Nel territorio di quella che l'Alberti definisce un'enorme pinacoteca, quale è l'Italia (Alberti 1951: 151), non ci sono solo le grandi e piccole città più note agli stranieri come Roma, Napoli, Venezia, Genova, Firenze, ecc., ma è possibile trovarci «centinaia di miniature e piccoli ed intimi medaglioni» (Alberti 1951: 151), tra cui Bitonto. Ci addentriamo dunque all'interno di questa città trapezoidale con al centro un'imponente "aquila»: la cattedrale di Bitonto. Definita come una tra le più belle chiese romaniche, nonostante $\mathrm{i}$ «danni» subiti dal Settecento, si presenta agli occhi dell'autrice come un'«aquila con una sola ala» (Alberti 1951: 151).

La descrive nei minimi dettagli e la paragona anche a chiese sparse per il mondo, per esempio a Parigi, a Praga o a Vienna, sottolineando che non sfigurerebbe in mezzo a monumenti più importanti (Alberti 1951: 151). Alla fine si pone una domanda, portando nuovamente tra le sue pagine il pensiero sull'uomo e la sua capacità di distruggere da una parte, tuttavia dall'altra ne esalta il suo saper immergersi nell'arte e la sua abilità nel costruire straordinari monumenti:

Questo dualismo insito nella sua natura umana accompagnerà l'uomo fino al termine della sua vegetazione terrestre? Vincerà mai una di queste metà? E se vincerà, quale delle due? II fango? Le ali dell'aquila? O almeno una sola? (Alberti 1951: 154).

166

Fabrica Litterarum Polono-Italica | 2019, nr 1 (1) 
Come delle aquile in volo puntiamo verso il cielo e oltre, perché la scrittrice vuole parlarci di Una stellina della via lattea. Questa stellina, un tempo chiamata Rubi, attualmente è nota come Ruvo. Ricca di storia e di ceramiche, grazie a degli attenti collezionisti, gli Jatta, è possibile ammirare nell'omonimo museo i vasi e le illustrazioni. Nel momento in cui Kazimiera Alberti entra nel museo, ha la sensazione che sia arrivata in una biblioteca piena di riviste illustrate, che altro non sono se non i vasi in ceramica conservati in esso. Da un quesito posto dall'autrice si evince che la visita al museo sia avvenuta nell'anno 1948: "Veramente ho passato questi brevi istanti nel XX⿳亠口冋 secolo, nell'anno 1948?» (Alberti 1951: 157).

Dopo l'ammirazione delle ceramiche e della cattedrale la città si spegne in un buio pesto che porta l'autrice ad essere un po' malinconica, seppur con un tocco di speranza, poiché guardando il cielo si rende conto di come le stelle brillino come se fosse estate e non novembre: «E forse resterà veramente dopo di noi qualche... stellina della Via Lattea» (Alberti 1951: 159).

Ci spostiamo giusto qualche chilometro più in là, per raggiungere una cittadina che ha avuto la possibilità di assaggiare la gloria imperiale: Andria (Alberti 1951: 161). L'Alberti scrive come l'epoca moderna abbia privato le piccole città dai grandi avvenimenti e come tutto ciò ora avvenga solo nelle capitali. Un tempo anche i piccoli paesi furono i protagonisti di fatti importanti, come nel caso di Andria, città a cui Federico II in partenza per le crociate affidò sua moglie Jolanda, che diede alla luce suo figlio, futuro erede al trono. Purtroppo dopo qualche giorno la donna morì per le conseguenze del parto e la città fu in lutto. La cripta della cattedrale di Andria, oltre alle spoglie di Jolanda, conserva anche le spoglie della seconda e della terza moglie di Federico II. Andria è I'unica città che è rimasta fedele all'imperatore e da qui la scrittrice prende spunto per poter riflettere sulla fedeltà non solo delle città, ma anche dell'uomo (Alberti 1951: 160-163).

Federico II di Svevia ha svolto un ruolo molto importante nella storia della regione pugliese. Ci sono tracce della sua presenza in tutto il territorio. II monumento più importante e che ci viene subito in mente pensando alla sua figura è proprio il Castel del Monte. ${ }^{1}$

La scrittrice ci suggerisce di leggere Un poema al vento dei secoli: Castel del Monte. Proprio per questo ci presenta la sua definizione di poema:

Quando diciamo «poema» per noi questo non racchiude solamente metrica di versi, composta in giambi, trochei od epodi da umane parole. Questa sarebbe una troppo povera definizione! Vi sono altri poemi, scritti con le minuscole note che assumeranno vita sotto l'archetto serafico di un violino o, elaborate dai più vari istrumenti, confluiranno nell'unità ideale sinfonica [...]. E poemi

1 Per approfondire l'argomento cfr. F. Cardini (2016).

167

Fabrica Litterarum Polono-Italica | 2019, nr 1 (1) 
furono scritti con l'esile trama degli arazzi fiamminghi o con i minuscoli vetri dei mosaici bizantini (Alberti 1951: 164).

Se da una parte per gli italiani la parola "poema» richiama inevitabilmente alla mente opere come l'Odissea, l'Iliade, dall'altra per i polacchi immediatamente l'associazione va all'epopea nazionale polacca, Pan Tadeusz. Ciò dimostra la grandezza e l'importanza che Kazimiera Alberti voglia donare all'immagine del Castel del Monte. Ne fa un'accurata descrizione da tutti i punti di vista, come se fosse una guida turistica, così da poter rendere questa presentazione allettante agli occhi del turista. Tuttavia l'attenzione della scrittrice non si sofferma soltanto sul "poema», ma ne esalta il personaggio che ha ideato il Castel del Monte, Federico II di Svevia:

Un uomo che visse tra le brume dello scandalo e tra le luci dell'entusiasmo: Federico II "stupor mundi», uno degli uomini più originali non solo della sua epoca ma di tutta la storia. [...] L'universalismo politico che egli sogna, l'unificazione dell'Europa non son forse idee che con tanta passione e dolore sommuovono la nostra generazione? (Alberti 1951: 164).

Siamo di fronte ad un imperatore dai mille interessi e dalle molteplici capacità, presentato come un precursore dell'arte del suo tempo in grado di valorizzare ed apprezzare opere artistiche capaci di brillare in eterno e di continuare a farlo in qualsiasi epoca. Di fatti l'Alberti definisce il Castel del Monte come la uprima culla del Rinascimento» (Alberti 1951: 165) seppur troppo riduttivo di fronte a cotanta bellezza architettonica. Si tratta di un poema al vento dei secoli, poiché nonostante tutte le intemperie, gli eventi storici, le epidemie resta sempre lì erto sul colle nella sua grande imponenza (Alberti 1951: 164-166).

Sono diverse le funzioni che questo monumento ha ricoperto nel corso dei secoli descritte anche dalla nostra personale "guida», Kazimiera Alberti: tenuta di caccia per l'imperatore, carcere per i suoi discendenti, rifugio durante un'epidemia, per poi diventare luogo prescelto da briganti e pastori con i propri greggi:

Il fato gli offre sempre nuovo destino nonostante tutto esso conserva la sua dignità e la sua originalità come se un incantesimo guardi l'intoccabilità della sua struttura (Alberti 1951: 166).

Tuttavia non ci sono testimonianze della presenza dell'imperatore di Svevia presso la tenuta. Attualmente ci sono tanti misteri e leggende che si celano dietro questo monumento tanto imponente ed importante, oltre a tutte le teorie legate alla forma ottagonale e la ricorrenza del numero otto e i suoi diversi significati. ${ }^{2}$

2 Per maggiori informazioni cfr. R. Astremo (2012); A. Lattanzi, N. Lattanzi (2015).

168

Fabrica Litterarum Polono-Italica | 2019, nr 1 (1) 
Lasciamo il misterioso Castel del Monte, «il poema più bello che Federico II abbia scritto in terra di Puglia» (Alberti 1951: 168), per poter dedicarci ad un altro momento di profonda riflessione storico-culturale. Canosa di Puglia, fondata da Diomede, è ricca di storia e leggende. In questa fermata il presente e le glorie del passato si intrecciano. Si respirano ancora nell'aria i tragici eventi della Roma antica, eppure all'improvviso la modernità irrompe lasciando spazio alla quotidianità canosina. È tempo di vendemmia e l'autrice osserva il paese in subbuglio per questa scadenza. Descrizioni realistiche e vivaci sui contadini e la loro uva, gli asini che spingono i carretti, riempiono le righe riguardanti questa cittadina. Ci rituffiamo nuovamente nel passato, grazie a delle iscrizioni romane, tradotte in italiano e commentate dalla scrittrice (Alberti 1951: 170-173).

Passando tra le vie lunghe e strette ci ritroviamo alle porte della città, laddove c'era la necropoli, che porta l'Alberti ad una riflessione sulla morte:

Dall'eternità dei tempi le tombe hanno interessato non solo la scienza ma anche l'umana fantasia. Dalle necropoli conosciamo la cultura. La morte fu sempre rispettata come la vita. Davanti la morte era inconcepibile ogni avarizia. Anche il più povero sacrificò l'eterno riposo qualche oggetto amato o qualche utensile. I ricchi portarono con sé buona parte della loro ricchezza [...]. La volgarità e la ferocia della vita hanno poi arso, rovinato, distrutto, rotto... ma la nobiltà della morte, la serietà e la sanità della tomba, intoccabile eredità secondo il codice non scritto, trasmesso da una generazione all'altra, con il loro sugello hanno guardato il segreto della loro ricchezza (Alberti 1951: 173).

La scrittrice coglie sempre l'occasione per invitare il lettore a visitare la Puglia, offrendogli diversi motivi per poterlo fare:

O straniero, se ti fecero tanto gran torto da battezzarti solo una volta nella vita, vieni a Canosa, ed il Battistero di S. Giovanni certamente ti ripagherà! (Alberti 1951: 175).

Riflessioni sul presente, il passato e le descrizioni terminano con un elogio di questo paesino:

E, sebbene pioviggini e sia assente quel meraviglioso scenografo che è il sole, Canosa, di faccia e di profilo, è per noi splendente, piena di luce interiore (Alberti 1951: 175).

Seguendo le antiche tracce dell'antica Roma, Kazimiera Alberti si prepara a visitare Canne. Tuttavia ci consiglia di fare una sosta con lei a Canosa, per poter ammirare l'Arco Romano. Lungo il tragitto da Bari a Canosa, la scrittrice osserva 
la Puglia e i pugliesi di notte. Ammira i contadini che lavorano negli uliveti e ne sottolinea la forza d'animo visibile attraverso il canto, nonostante i problemi che possano affliggerli riguardanti il lavoro, il loro raccolto, ecc. (Alberti 1951: 176-177).

L'Arco romano non le riporta a galla scene di guerra, brutti ricordi, quelle terribili sensazioni che gli altri archi avevano suscitato in lei. Al contrario, questo umile e sobrio arco, immerso nella campagna, sembra far parte del paesaggio ed è insito in un senso filosofico, piuttosto che in un senso bellico:

Tutti gli altri erano, si può dire, ... urbani, nel complesso di pietra, sullo sfondo del rumore stradale. Questo qui è... agreste, con i suoi piedi fortemente piantati nella fertile terra (Alberti 1951: 178).

Sedendo su di una pietra, appoggiata con le spalle all'arco, l'autrice si domanda come saranno giudicati i trionfi del passato. In conclusione l'attenzione è posta sulle generazioni future, per le quali la scrittrice si domanda se nella loro "televisione» apparirà il ricordo del contadino, che passa per l'Arco di trionfo, che lavora e canta nei suoi campi, per poter assolvere il XX secolo (Alberti 1951: 179).

Proseguiamo il nostro viaggio sulla scia degli eventi storici, seguendo le tracce di Annibale. Piuttosto che descrivere solo gli eventi storici, attraverso la battaglia di Canne, la scrittrice cerca di capire il senso di questi eventi e le lezioni di vita che ne susseguono. Come sottolinea tra queste pagine, il suo pensiero riguardo alle guerre è ben saldo:

Da bambini abbiamo giocato sul grembo della prima guerra mondiale e la seconda ci ha baciato tragicamente in fondo al cuore. Noi siamo la generazione per la quale la parola "guerra» non è «gloria», ma solamente «dramma» (Alberti 1951: 179).

Ci ritroviamo dunque ad una lezione all'aria aperta, direttamente sul campo:

Ci interessa oggi la psicologia dell'aggressione e le sue conseguenze. In nessun luogo la studierai tanto bene come su questo campo, oggi. [...] La lezione fu semplice: questo compito di storia fu scritto da centoventimila uomini, da un mattino ad una sera. Naturalmente lo scrissero con l'inchiostro rosso delle proprie vene (Alberti 1951: 180).

Ci spiega come l'aggressività non faccia altro che generarne dell'altra e Canne ne è l'esempio per eccellenza. In questo caso ne approfitta per sottolineare come purtroppo la lezione non fu appresa dall'umanità per evitare che tutto ciò si ripetesse nei secoli successivi, ma è stata purtroppo solo spunto per poter sfruttarne 
le sue strategie. Ci illustra come quella di Canne non sia una semplice battaglia tra due popoli, bensì:

Canne è qualcosa di più. È la ferita che tragicamente si ripete e si riapre ad ogni generazione, su tutto l'orbe terraqueo. E se l'umanità non guarirà in qualche modo questa ferita, certamente non creerà mai una più alta cultura. Una cultura basata sulla giustizia ed eguaglianza tra i popoli (Alberti 1951: 181).

Per l'autrice una maggiore comprensione della battaglia sarebbe possibile se fosse descritta, commentata e illustrata nei libri scolastici, seppur in maniera completamente diversa rispetto a quella versione che c'era nei libri della sua epoca (Alberti 1951: 181).

Ancora una volta, la battaglia ha portato a galla il ricordo della guerra, che cerca di superare, portandosi con sé il ricordo di Canne, come il «campo di una celebre battaglia antica» (Alberti 1951: 182), un luogo non solo archeologico, ma con una morale che deve spingere gli animi verso il pacifismo.

Nella prossima tappa riappare ancora una volta l'imperatore di Svevia, Federico II, che durante le crociate verso la Terrasanta, passando per Altamura, decise di lasciare lì i suoi uomini stanchi e ammalati. Con gran sorpresa al ritorno ritrovò i suoi soldati più forti di prima:

Federico, monarca munifico, non fu sconoscente. Scelse, da poeta qual era, un poetico nome per la città, quarta nella propria storia, nuova per i tempi moderni: "Altamura». La munì di castello e di mura, e fondò in essa quella meraviglia dell'arte romanico-pugliese che è il Duomo (Alberti 1951: 185).

Prima di proseguire per il nostro viaggio in terra di Puglia, l'autrice ci parla della leggenda della città di Altamura legata all'amico di Ercole Filottete. Nella storia di questa cittadina è ricorrente il fatto che ci sia un'aria pura, salubre. Difatti il primo nome della città fu Petilla, la "rosa selvatica" (Alberti 1951: 184). Ciò nonostante fu rasa al suolo da Annibale e successivamente abitata da Romani, Greci e poi Ostrogoti. II paesaggio colpisce molto Kazimiera Alberti, però quello che cattura maggiormente la sua attenzione è la cattedrale di Altamura che, con il suo imponente e artistico portone, lascia la nostra «guida» stupefatta.

Seguendo la nostra «cicerone» proseguiamo nell'entroterra pugliese per raggiungere Gravina. In questa località l'Alberti passa la parola ad un venditore ambulante, che ci guiderà e ci presenterà quello che di bello questa cittadina ha da offrire: dai musei alla chiesa di San Sebastiano, dalla Cattedrale al monumento funerario di Angelo Castriota, e così via... II venditore oltre ai soliti monumenti, sa soddisfare anche le esigenze dei turisti che non si sanno accontentare, raccontan-

171

Fabrica Litterarum Polono-Italica | 2019, nr 1 (1) 
do aneddoti e «segreti» del posto. Questo personaggio un po' stravagante mostra Gravina attraverso i monumenti, i quadri, i santi:

In un angolo - guardate bene! - l'affresco dell'elemosina di S. Martino. Sul cavallo bianco il salto taglia con la spada il suo largo mantello, di cui il povero sostiene la falda. La figura di S. Martino, vigorosamente rilevata, rappresenta un secolo più progredito nell'arte pittorica, bel prodotto della pittura del $\mathrm{XV}^{\circ}$ secolo (Alberti 1951: 189).

Il venditore ambulante dialoga con il lettore e gli dà la possibilità di scegliere quello che preferisce. Propone tutti gli stili e i monumenti da scegliere in base ai propri gusti personali. È un ambulante colto e completo, che fa riferimento a Federico II, il quale ha definito Gravina "giardino delle delizie» (Alberti 1951: 190) per la bellezza dei suoi paesaggi tutti da ammirare. Ci coinvolge e ci racconta curiosità della città con tono giocoso e divertente:

Peccato che la malaria nel 1623 costrinse gli abitanti a prosciugare il lago artificiale che si estendeva nei pressi della città. Se no, potrei mostrarvi un altro articolo: le cicogne. Fino a quell'epoca, Gravina, unica città d'Italia, fu sede prediletta delle cicogne, che protette da nobili e popolo, nidificavano su ogni tetto. Ora purtroppo l'articolo è esaurito... (Alberti 1951: 190).

È interessante notare come il commerciante si soffermi sugli eventi storici, sulla presentazione dei monumenti e degli elementi architettonici e naturali, senza tralasciare il folklore dell'epoca, con la descrizione di matrimoni e dei costumi tradizionali:

Se siete amanti del calore locale, forse solo qui, in tutta la provincia di Bari, disponiamo di folklore. Venite ad assistere ad un matrimonio o ad una festa locale. Vedrete uomini e donne sfoggiare i loro severi costumi tradizionali che qui ancora si conservano non come cimeli da museo, ma come parte attiva della vita (Alberti 1951: 191).

Sereni e spensierati dopo questo lieto incontro a Gravina, siamo pronti a spostarci verso la nostra prossima fermata: Conversano. Arrivati in questo paesino è come se ci fosse lo scontro di due forze contrastanti: il fenomeno husserliano ed il realismo.

Kazimiera Alberti ci fornisce una spiegazione di questa fenomenologia husserliana riguardante l'esistenza umana, che l'ha da sempre incuriosita, di come alcune persone con delle stesse caratteristiche possano nascere e crescere in un determinato luogo. È come se la tradizione, la natura e il luogo influenzassero la personalità

172

Fabrica Litterarum Polono-Italica | 2019, nr 1 (1) 
di un uomo. Partendo da questo presupposto, ci presenta diversi personaggi che hanno reso interessante e talvolta fuori dal normale la storia di Conversano:

Tancredi, figlio di Goffredo, II $^{\circ}$ conte di Conversano, eroe crociato che tanta parte occupa nella "Gerusalemme Liberata». [...] Con l'avvento della casa Acquaviva d'Aragona, Conversano cominciò a produrre i tipi più forti del suo repertorio (Alberti 1951: 193).

Uno dei personaggi più famosi ed estrosi della storia pugliese è proprio Giangirolamo II d’Aragona, noto come il "Guercio di Puglia». Quest'ultimo, oltre a far squagliare vivi dei canonici ed a rivestire le poltrone del proprio castello con le loro pelli, è celebre per aver commissionato il più grande ciclo pittorico della Gerusalemme Liberata, ma soprattutto per essersi inventato un modo per eludere le tasse imposte dal re del regno di Napoli Ferdinando I d'Aragona per la nascita di nuovi centri urbani. ${ }^{3}$ Grazie a questo stratagemma inventato dal Guercio di Puglia, sono nati i trulli di Alberobello, oggi patrimonio dell'UNESCO a partire dal 1996.

Oltre ai diversi personaggi bizzarri che si concentrano nella storia di Conversano, degni di nota sono anche i monumenti ed il paesaggio:

Così, con la borsa piena, ripartiamo da Conversano, allegri per essere riusciti ad "accendere due candele insieme» (come dice il proverbio polacco) davanti a due idee che non si possono sopportare vicendevolmente. Davanti la fenomenologia di Husserl e, contemporaneamente, davanti l'onesto e vieto realismo (Alberti 1951: 197).

Seguendo l'autrice ci ritroviamo ad ammirare le grotte di Castellana, definite come un "castello sotterraneo della Puglia» (Alberti 1951: 198). Confrontandole con le grotte che ha visitato in giro per il mondo, ci propone di visitarle come se fossero i castelli della Loira, le chiese gotiche francesi ed in questo modo:

Vedrai un teatro nel quale non fosti mai; davanti ai tuoi occhi si apriranno scenari mai veduti, paurosi e capricciosi, armonici e mostruosi, primitivi e raffinati, forti e delicati! (Alberti 1951: 199).

Ci anticipa in qualche modo le sensazioni che ci susciterà la vista di queste grotte che ci lasceranno a bocca aperta:

Sarai sbalordito davanti ad un'architettura mai gustata nella vita; architravi, capitelli, arcate, cripte, cupole; ed allora comprenderai che la pietra vive,

3 Vedi R. Astremo (2015).

173

Fabrica Litterarum Polono-Italica | 2019, nr 1 (1) 
che questa pietra creduta insensibile ha la forza di creare migliaia di aspetti e visioni con genio folle da gigante. Comprenderai che questa pietra è da sola architetto e scultore, che, dal travaglio dei secoli, trae dal suo interno le forme dell'uomo stesso non presentite, e vedrai, con tutto il tuo orgoglio di originale, quanto sia grande la tua piccolezza, quanto enorme la tua inferiorità, davanti la fantasia e la forza della natura (Alberti 1951: 199-200).

È un elogio alla natura, in grado di creare nel corso dei secoli uno scenario spettacolare che non si può assolutamente perdere:

Occorre solo scendere e guardare. II resto lo farà da solo questo magico castello sotterraneo nel quale tu visitatore, col tuo gran talento artistico, con la tua fantasia e la tua individualità, diventi un pigmeo (Alberti 1951: 200).

La nostra fermata successiva è la città dei trulli, Alberobello. L'invito che ci fa questa volta è quello di abbandonare l'idea della magnifica architettura delle città italiane più note, per poter osservare da più vicino lo stile preistorico. Qui, «nella culla dell'architettura» (Alberti 1951: 202), l'Alberti fa un inno dedicato alla pietra:

Vieni qui ad Alberobello e qui, in questa culla dell'architettura, comprenderai quanto amore e rispetto per la pietra, nel suo testamento, ha lasciato il primitivo architetto. Per primo egli cambiò la pietra in suo protettore, ché gli desse riparo e sicurezza davanti gli uragani ed i cicloni, e ricovero dopo il lavoro sotto l'ardente sole (Alberti 1951: 202).

I trulli erano diffusi già nell'epoca preistorica in Valle d'Itria, soprannominati tholos, utilizzati per seppellire i morti. Tuttavia i trulli che attualmente ritroviamo ad Alberobello sono quelli risalenti al XIV secolo, periodo durante il quale il territorio alberobellese era sotto il dominio dei Conti di Conversano. ${ }^{4}$

Passeggiando tra le vie di questa cittadina sembra che qui la vita fosse più tranquilla, che l'uomo che ci viveva tanto tempo fa fosse sereno e più felice. L'autrice ci fa una piccola lezione sullo stile architettonico di queste case dove è possibile ammirare:

[...] il più crudo primitivismo, questa lettera «A» nell'abicì dell'architettura, che ha racchiuso in sé tutti gli elementi strutturali delle altre lettere. L'analfabeta più difficilmente impara questa prima lettera... tutte le altre sono poi più facili. Guarda, prendi ad Alberobello la tua prima lezione; poi comprenderai più facilmente ogni geroglifico architettonico. [...] Guarda il progetto dell'architetto

4 http://www.italia.it/it/scopri-litalia/puglia/poi/la-storia-dei-trulli-di-alberobello.html. Accesso: 11.02.2019.

174

Fabrica Litterarum Polono-Italica | 2019, nr 1 (1) 
preistorico! Ecco il rifugio che egli costrui per i suoi vivi e per i suoi morti. Di qui è partito il primo filo per Creta, Micene e la Grecia (Alberti 1951: 203).

Ancora una volta, anche in questa località, ci pone davanti ad un quesito nato da una riflessione sulla vita moderna rispetto a quella del passato. Sembra che alla sola vista i trulli diano la possibilità di essere sereni e felici, al contrario delle costruzioni moderne, come ad esempio i grattacieli, che non sono in grado di regalarci le stesse emozioni positive (Alberti 1951: 204).

Per presentarci il capoluogo salentino, Lecce, la scrittrice decide di portarci indietro nel tempo fino all'epoca barocca, per poter comprendere a pieno lo stile di questa città che, al contrario della maggior parte delle altre, ne vanta uno ben definito: quello barocco per l'appunto.

Per rendere al meglio la descrizione di questa località, paragona le città agli uomini e ci dimostra «come gli uomini, le città nascono sotto qualche stella buona o cattiva» (Alberti 1951: 206) e ripercorre così tutte le fasi della nascita di questo gioiello barocco. Parlando di questo stile prorompente, ne descrive gli angeli allegri e vigorosi, proponendoci di bere un bicchiere del buon vino di Lecce (definito dall'autrice come uno dei migliori vini che abbia mai bevuto) e presentandoci alcune delle tante chiese costruite con la particolare e fragile pietra leccese (Alberti 1951: 207-208).

È come se il centro storico di Lecce sia rinchiuso tra le mura, in una sorta di bolla capace di fermare il tempo:

Ogni volta, quando da una delle tre porte usciamo nella città moderna, abbiamo sempre l'impressione di risvegliarci in un'altra epoca. E ci riesce difficile credere di essere nel XX secolo, poiché qui tutto è tranquillità e silenzio (Alberti 1951: 209).

Nella città in cui tutto sembra essere fermo all'epoca barocca, solo gli abiti dei leccesi dimostrano che stanno visitando la città dopo la seconda guerra mondiale.

Con la lucerna ad olio nelle mani... giungiamo insieme ai coniugi presso Massafra. Qui l'Alberti e Cocola si trovano di fronte a un paesaggio suggestivo, con un'aria bizantina. La Tebaide d'Italia, utilizzato dalla scrittrice come guida per visitare questa città, è un testo di uno storico nato in questa terra, Vincenzo Gallo, al quale è dedicato anche il capitolo. Il segreto che ci presenta tra queste pagine è quello di un villaggio bizantino formato da tante grotte vicine, poco accessibili, ideale per i cristiani che cercavano di rifugiarsi e praticare la propria religione, Iontano dagli occhi dei Romani (Alberti 1951: 210-211). Con in mano una lanterna ad olio, iniziamo l'esplorazione di queste meravigliose cappelle bizantine. L'autrice

175

Fabrica Litterarum Polono-Italica | 2019, nr 1 (1) 
vuole farci capire che non esiste solo una forma di «bizantinismo», poiché possiamo distinguerne uno più ricco ed uno più povero:

Hai sempre pensato che il «bizantinismo» sia Ravenna, S. Vitale e S. Apollinare, S. Giovanni ed il Sepolcro di Galla Placidia, i mosaici dai fantastici colori, i ricchi capitelli, le cupole maestose, gli alti campanili. Certamente questo è «bizantinismo»! Ma l'ufficiale, il ricco, quello della capitale, della sfera che governa, che ha a sua disposizione centinaia di svariati architetti ed artisti. Ma vi è ancora l'altro bizantino, povero, lavorato dalla mano e dalla fantasia di fanatici eremiti, disseminato in tutta la Puglia, che ha raggiunto la sua espressione concentrata nella «Tebaide d'Italia»: Massafra (Alberti 1951: 213).

Oggetto centrale di queste pagine è proprio la lanterna, simbolo di un'antica festività bizantina in cui la leggenda narra di una festa dell'illuminazione soprannominata "Festa del Paradiso», dove durante la notte gli abitanti delle grotte si dirigevano giù, nella vallata, con in mano una lume ad olio fra le mani (Alberti 1951: 214). Proprio riflettendo su questa leggenda e le luci moderne, la scrittrice spera che la lanterna ad olio possa illuminare il sentiero della vita di ognuno di noi (Alberti 1951: 215).

Dopo aver posato le nostre lucerne in quel di Massafra, siamo pronti ad ammirare le antiche mura megalitiche conservate in quel di Manduria, città nota per i fichi secchi. L'autrice ripercorre un po' i tratti salienti della storia e della costruzione delle mura di questo paese. Ci menziona la leggenda legata all'acqua, nella caverna, dove probabilmente in precedenza si trovava proprio il centro della città antica. È suggestivo come a seconda delle origini dei fondatori, il nome della città possa assumere due significati completamente opposti: Città forte oppure Buon Augurio (Alberti 1951: 218). Tuttavia l'Alberti stessa ci sottolinea come nella storia queste due definizioni siano del tutto contrastanti e in disaccordo.

La nostra prossima stazione, Giurdignano, è la zona in cui si concentra il maggior numero di dolmen e menhir. Ciò porta la nostra "guida polacca" a definirla come un "giardino megalitico" (Alberti 1951: 219). Proprio mentre è in questo giardino, immersa nei suoi pensieri, ancora una volta tende a difendere la Puglia da quelle persone ignoranti che, come ella stessa ci ricorda nell'Introduzione, non sanno riconoscere la differenza tra un dolmen ed un menhir (Alberti 1951: 5). Sulla base di questo, decide di scrivere una cartolina, in cui ancora una volta esorta il lettore a visitare questa terra ed ammirarne le meraviglie. Ribadisce il fatto che ritenga questa una regione per visitatori molto intelligenti ed aperti mentalmente (Alberti 1951: 220).

Dalla provincia di Lecce, giungiamo a nord della Puglia e ci ritroviamo nella Capitanata, precisamente a Foggia. L'Alberti ci parla dello stemma con le tre lingue di fuoco, per poterci introdurre la leggenda della Madonna dei sette veli, ritrovata

176

Fabrica Litterarum Polono-Italica | 2019, nr 1 (1) 
da dei pastori che seguirono queste tre fiammelle di fuoco e ritrovarono in uno stagno l'icona avvolta appunto da sette veli. Pare che proprio lì dove fu ritrovato il quadro della Madonna sia stata costruita la cattedrale di Foggia. Ci racconta come nei secoli i veli siano stati spostati solo una volta nel Cinquecento, poiché un vescovo aveva paura che si potesse celare un'immagine diabolica dietro di essi. Fu l'unica volta in cui fu aperta. Da quel momento ognuno ha avuto la possibilità di poter immaginare il volto della Madonna in maniera del tutto soggettiva e questo alletta molto la scrittrice che ritiene che gli occhi colgano le cose a loro piacimento (Alberti 1951: 221-223).

Restiamo ancora a Foggia, per poter ripercorrere insieme un po' di storia legata ancora una volta ad un personaggio molto importante: Federico II di Svevia. II rapporto tra questa città e l'imperatore è sempre stato un tira e molla, un odi et amo. L'autrice ci ricorda come lo "stupor mundi», sia riuscito ad entrare nella cittadina di Foggia, che precedentemente gli aveva negato l'accesso, grazie alla sua poesia (Alberti 1951: 224-225). Ci racconta come:

Foggia ebbe la strana fortuna di essere egualmente amata da Federico II e dal suo più accanito nemico, Carlo d'Angiò. Nella sua Cattedrale trovarono riposo il cuore di Federico e le visceri di Carlo. Queste visceri che tanto spesso avevano ribollito di collera solo al ricordo del nome svevo. Così, in questa strana forma, la storia inscenò il perdono evangelico dopo la morte. Un bel gesto da parte sua. Ma la natura, giudice più spontaneo della storia, [...] decise un altro giudizio. II grande terremoto del 1731 distrusse quasi tutta Foggia (Alberti 1951: 226).

Quindi ci ritroviamo in una terra che fu distrutta dal terremoto nel lontano 1731 e luogo in cui le reliquie di due sovrani, che tanto hanno apprezzato questa località, furono perdute. Purtroppo non solo la natura aveva in serbo delle sorprese "distruttive" per questa città. Durante la seconda guerra mondiale, Foggia, fu bombardata e devastata (Alberti 1951: 226).

Dopo averci parlato di Foggia, siamo pronti per perlustrare e approfondire cosa ci può offrire la città di Troia. Sin dall'inizio l'autrice ci sottolinea come il nome di questo paese ci porti inevitabilmente a pensare al poema dell'Odissea. Inizia qui a raccogliere gli elementi che ci sveleranno il motivo per cui questa cittadina abbia questo nome. Ad esempio ci parla della vicende di Diomede, fondatore di diverse città pugliesi, che ritrovando la propria moglie con un altro uomo decise di abbandonare lei e la sua terra. Fondò una città dal nome Ecana, lì dove conobbe la donna della sua vita Ecania. Purtroppo la posizione di questo paesino, sulla Via Appia, ha fatto sì che fosse luogo di guerre e spargimenti di sangue nel corso dei secoli. Tuttavia è una località che non si arrende mai (Alberti 1951: 228-232). 
Ecana, dopo essere stata cosparsa di sale, grazie alla sua tenacia è stata in grado di reincarnarsi in Troia. La scrittrice ci propone le due ragioni che hanno dato vita a questo nome:

[...] forse furono riuniti insieme questi due simboli, il poetico ed il materiale. Diomede trasmise la gloria troiana, la scrofa realizzo le sue mute promesse, superbamente pingui. Il villaggio si sviluppò. Divenne florido, nell'unto della carne e della gloria (Alberti 1951: 232).

Andando avanti, ma restando sempre nella stessa zona, l'esule polacca ci parla di un ordine del 1093 soprannominato la "Tregua di Dio», ovvero una sorta di pausa, di tregua, per poter recuperare i cadaveri e seppellirli con dovuto rispetto. Ciò fa sorgere nell'Alberti un senso di disappunto per quello che è avvenuto a Varsavia durante la rivolta del 1944 e soprattutto per quanto riguarda la "raccolta» dei caduti per le strade della capitale polacca (Alberti 1951: 234).

Troia era una di quelle città che si oppose alla consegna delle proprie chiavi all'imperatore svevo, Federico II. Iniziò una sorta di gioco tra i cittadini ed lo "stupor mundi», che ovviamente non andò a finire nel migliore dei modi ed ancora una volta Troia fu rasa al suolo. Tra i monumenti ce n'è uno che ci può dimostrare tutti i volti di questa località:

[...] tutte le reincarnazioni di Troia, illustri e funebri, dolorose e luminosi si centralizzeranno nella Cattedrale, le di cui pietre e marmi si ricordano ancora dai tempi di Ecana. E l'asimmetrica rosa della facciata, unica nel suo stile in tutta la Puglia, con le transenne l'una differente dall'altra, sembra simbolizzare le varie e differenti incarnazioni storiche della città (Alberti 1951: 237).

Di qui Kazimiera Alberti coglie i pregi che la Cattedrale offre al viaggiatore e ne descrive la facciata trattata come "la parte più importante di questa "Sinfonia", che dopo una più intima conoscenza la si può denominare "Patetica"» (Alberti 1951: 238), il rosone e le porte definite dai critici dell'arte come "le più preziose dell'Italia meridionale dopo quelle della Cattedrale di Benevento» (Alberti 1951: 239).

Continuiamo la nostra gita all'insegna della scoperta dei segreti pugliesi, fermandoci questa volta a Lucera. II passato e le leggende sono sempre presenti, con un tocco di modernità, come la tecnica del fotomontaggio:

[...] i banchieri furono insospettiti dal fatto che l'originale moneta con lo stesso elmo di Minerva e la lettera "L» fosse infilata in una vetrina di museo. Dissero che questo montaggio storico era mal riuscito (Alberti 1951: 239).

178

Fabrica Litterarum Polono-Italica | 2019, nr 1 (1) 
Successivamente la scrittrice rintroduce tra le righe Federico II, colui che ha trasformato la Puglia rendendola esotica, portando a Lucera un gran numero di saraceni per renderli più «europei» africanizzando così invece la cittadina (Alberti 1951: 245-247).

L'autrice inizia poi una descrizione del paesaggio incantevole ricco di colori, che lei vorrebbe saper riprodurre su una tavolozza. Si ripresenta anche qui, il dispiacere che prova per la malvagità umana in grado di abbattere ogni cosa: "Strano animale è l'uomo! Con gioia infantile costruisce, ma con voluttà distrugge!» (Alberti 1951: 249).

Parlandoci dell'Anfiteatro di Lucera e tornando un po' nel passato, inizia una riflessione riguardante il tempo dell'uomo moderno e dell'uomo del passato:

L'uomo contemporaneo si è formato uno stile di vita che divora tutto il suo tempo. L'uomo si è impoverito, ha perduto il suo prezioso gioiello: il tempo! Non ha tempo per niente. Né per pensare né per riposare, né per studi più profondi né per il divertimento. Tutto il mondo si affretta troppo [...]. Da questo punto di vista l'uomo dell'antichità batte l'uomo contemporaneo. Egli aveva il tempo per tutto. Non il tempo governava lui ma lui il tempo (Alberti 1951: 253).

Questo ci dimostra come l'uomo contemporaneo già negli anni Cinquanta fosse più "povero» di tempo rispetto a quello dell'antichità, pur avendo a disposizione molti più comfort e mezzi che avrebbero dovuto facilitargli la vita.

Per renderci al meglio questa località che è tanto piaciuta alla nostra "guida», decide di paragonarla ad una giovane donna che ha solo 77 anni (Alberti 1951: 254). Vede la città come un luogo semplice, naturale e modesto:

La venere di Lucera vive, là dove è nata! Ha resistito a tutte le mode della linea femminile. II gotico, il rococò, la «maschietta». [...] Sono passati i secoli, ma la Venere trovata nelle Terme brucia, infiammata dalla forma eternamente attuale, delle proporzioni classiche, che entusiasmano tutti e non invecchiano mai (Alberti 1951: 255).

Attraverso l'interpretazione di un'iscrizione del Museo Lucerino nota come i periodi bui ci siano sempre stati in tutte le epoche e che quella contemporanea sia molto più caotica e movimentata rispetto all'antichità (Alberti 1951: 255).

In questo nostro cammino con Kazimiera Alberti, facciamo una sosta presso Castel Fiorentino, luogo dietro cui si cela una leggenda riguardante una meteora, che secondo una profezia, terminò il suo viaggio su dei fiori. Come già l'autrice anticipava all'epoca, sulla base delle idee del tanto acclamato Federico II, prevedeva che ci potesse essere un universalismo poetico europeo (Alberti 1951: 256).

179

Fabrica Litterarum Polono-Italica | 2019, nr 1 (1) 
Mette in risalto il genio dello "stupor mundi», colui che propose l'unità dell'Europa, quell'uomo che non è solo un semplice imperatore: "Ma vi fu anche un Federico - filosofo ed un Federico - poeta» (Alberti 1951: 256). Castel Fiorentino è il luogo in cui l'imperatore si è spento nel 1250. La profezia di Michele Scotto si è realizzata: "Morirai presso una cortina di ferro, nel luogo che ha il fiore nel suo nome» (Alberti 1951: 258).

Una domanda sull'Europa ci porta a riflettere sul destino di quest'ultima e non solo:

Forse che l'Europa dovrà divenire tra secoli un pugno di rovine, come Castel Fiorentino? Od invece questo nome coronerà l'idea di Federico non imperatore, ma filosofo e poeta? L'Europa unificata? O «Castel Fiorentino»? (Alberti 1951: 259).

Siamo giunti al capolinea e concludiamo la nostra avventura in Puglia insieme alla nostra "cicerone» con un Brindisi di addio, fatto non con del buon vino pugliese, esaltato nelle pagine precedenti, ma con un cincin con l'acqua dell'Acquedotto Pugliese. Qui I'Alberti si concentra, su quest'ultimo, poiché si tratta di un'infrastruttura che ha dato realmente una svolta a questa regione. Di qui inizia una riflessione, sull'acqua che non sempre viene apprezzata e confeziona quasi una campagna pubblicitaria contro il suo spreco. La costruzione dell'Acquedotto ha coinvolto tante persone e l'autrice decide di seguire il percorso che ha portato alla sua realizzazione. Questo è il segreto più profondo della Puglia, così presentato dall'autrice:

Ecco l'ultimo "Segreto di Puglia». Forse il più interessante, certo uno dei più emozionanti e vitali. Il segreto della rinascita, il segreto della vita, il segreto dell'avvenire, il segreto del lavoro costruttivo umano... (Alberti 1951: 273).

In questo caso, il lavoro dell'uomo ha offerto un elemento davvero importante, dimostrando che egli non è solo in grado di distruggere. È un inchino di fronte all'intelligenza dell'uomo in grado di tale maestria. Un elogio al lavoro, alla sorveglianza e a tutto quello che serve per poter far funzionare l'acquedotto.

L'ultimo invito che ci fa è quello del nostro brindisi per congedarci:

Voglio offrirti acqua dell'acquedotto pugliese. Con essa berremo il brindisi di addio. Prendi nelle mani questo bicchiere di «vilissima rerum», qui, all'ombra, presso questa fontana. [...] Bevi con me questo bicchiere d'acqua, alla gloria del cervello e della tenacia, del sacrificio e dell'energia umana! lo riempirò ancora di essa la bottiglia che mi rinfrescherà durante il viaggio. [...] ogni ricchezza che ho visto su questo mondo aveva sempre, nascosto nel suo in-

180

Fabrica Litterarum Polono-Italica | 2019, nr 1 (1) 
timo, bacilli e gocce di veleno, [...] almeno oggi sono sicura che questa mia, modesta odierna, è pura (Alberti 1951: 274-275).

Così termina il nostro viaggio, con una speranza, con un brindisi fatto con la pura acqua proveniente dall'Acquedotto Pugliese.

Kazimiera Alberti è un'autrice polacca che ha vissuto in Italia e si svela attraverso le sue opere. Una donna che ha reagito alla bufera bellica, attraverso la ricerca della bellezza dei paesaggi, monumenti, cultura e folklore che l'Italia e più nello specifico il Sud Italia è in grado di offrire.

L'esule polacca non si concentra sulle questioni sociali e politiche, d'altra parte descrive tutta la sua ammirazione per questa terra baciata dal sole, in grado di contenere tanti segreti. Segreti che la scrittrice decide di condividere solo in parte con il lettore, invitandolo a visitare questa splendida terra. Una regione che secondo l'autrice è stata capace di progredire e di svilupparsi. Difende a spada tratta questo territorio dagli scettici e dagli ignoranti, considerandolo all'altezza di altre regioni, come per esempio la Toscana.

Tema centrale è la rivendicazione della bellezza, che ritroviamo come filo conduttore nella maggior parte dell'opera. A prevalere tra le pagine non sono tanto i paesaggi, quanto i monumenti. L'autrice si sofferma su dettagli che possono risultare apparentemente superflui, come l'asinello o la vita che si svolge per le strade, tuttavia sono proprio questi particolari a mostrare la realtà meridionale e il suo folklore. Senza mai dimenticare il genio dell'uomo e la sua capacità nel costruire.

I paragoni a livello culturale, paesaggistico e geografico, che l'Alberti fa nelle sue opere, ci fanno comprendere che si tratta di una donna che ha viaggiato molto, che ha una profonda conoscenza del mondo, dando quindi una maggiore credibilità alle sue affermazioni.

Il ricordo della guerra è ancora vivo nella sua memoria, infatti il travelogue è ricco di passaggi in cui i ricordi della bufera bellica vengono fuori, per una grotta che ricorda il bunker in cui ci si rifugiava quando le sirene iniziavano a suonare; un pregiudizio dei Francesi nei confronti degli Italiani la porta a pensare che le discriminazioni possano comportare, come nel caso della seconda guerra mondiale, uno sterminio di massa.

Già all'epoca si rendeva conto che era necessaria un'Europa unita proprio come si evince dal pensiero di Federico II. È una viaggiatrice consapevole e curiosa, e attraverso un linguaggio semplice e diretto ci regala delle descrizioni ricche di metafore, che rendono scorrevole la lettura del volume. Nonostante quello che le è successo continua a nutrire delle speranze nella sua vita.

Nell'ultima parte del testo presenta delle novità, introducendo il miracolo dell'acqua: I’Acquedotto Pugliese, il più grande segreto della Puglia appunto. Grazie

181

Fabrica Litterarum Polono-Italica | 2019, nr 1 (1) 
all'acquedotto la scrittrice dimostra il dualismo dell'uomo (tema ricorrente nel libro) che non è solo in grado di distruggere tutto quello che lo circonda, ma è anche capace di costruire qualcosa di utile.

II reportage di viaggio si conclude con un congedo da questa terra che è stata in grado di risollevare il suo animo, pur trattandosi di un addio solo letterario, dato che la scrittrice vivrà e poi si spegnerà a Bari.

Kazimiera Alberti testimonia l'effetto che questa terra ha avuto sulle sue opere e sul suo animo. Attraverso i suoi occhi giunge a noi una descrizione senza pregiudizi, che al contrario di altre tende a smontare i luoghi comuni del Sud Italia.

\section{Bibliografia}

AlbertI Kazimiera (1951): Segreti di Puglia. Traduzione di A. Cocola. Napoli.

AlberTI Kazimiera (2007): L'anima della Calabria. Traduzione di A. Cocola. Soveria Mannelli.

Astremo Rossano (2012): 101 misteri della Puglia che non saranno mai risolti. Roma (e-book).

Astremo Rossano (2015): II trullo siamese e una storia di quotidiana gelosia. In: Idem: 101 storie sulla Puglia che non ti hanno mai raccontato. Roma.

CARDINI Franco (2016): Castel del Monte. Bologna.

GIULIANI Francesco (2009): "Viaggi novecenteschi in terra di Puglia". Nicola Serena di Lapigio - Kazimiera Alberti - Cesare Brandi. Foggia.

JANAS Janina (2007): Szkice o literaturze i języku polskim. Fasano.

LATTANZI Antonella, LATTANZI Natalino (2015): Guida insolita ai misteri, ai segreti, alle leggende e alle curiosità della Puglia. Roma (e-book).

\section{Abstrakt}

\section{Apulia, region do odkrycia. Podróż z Kazimierą Alberti}

Celem artykułu jest prezentacja utworu Kazimiery Alberti Segreti di Puglia (Tajemnice/ Sekrety Apulii), który został opublikowany w ramach drugiego tomu serii zatytułowanej Italia celebre e sconosciuta (Włochy Znane i Nieznane). Podążając za Alberti, odkrywamy w jej relacji z podróży niektóre sekrety ziemi apulijskiej mało znane nie tylko cudzoziemcom, lecz również włoskiemu czytelnikowi. Autorka zachwyca się architekturą, zabytkami, krajobrazem apulijskim, ponadto przedstawia w szczegółach życie codzienne,

182

Fabrica Litterarum Polono-Italica | 2019, nr 1 (1) 
zwyczaje, tradycje, a także po mistrzowsku wprowadza czytelnika w historię regionu, wzbogaconą o mity i legendy. Utwór Kazimiery Alberti jest doskonałym zaproszeniem do odkrycia tego fascynującego, choć mało znanego regionu.

Słowa kluczowe: Kazimiera Alberti, Apulia, Włochy, reportaż z podróży, Segreti di Puglia 